\title{
ROLE OF GENERALIZED COSMIC CHAPLYGIN GAS IN ACCELERATING UNIVERSE : A FIELD THEORETICAL PRESCRIPTION
}

\author{
Prabir Rudra ${ }^{1, *}$ \\ ${ }^{1}$ Department of Mathematics, Bengal Engineering and Science University, Shibpur, Howrah-711 103, India. \\ Department of Mathematics, Pailan College of Management and Technology, \\ Bengal Pailan Park, Kolkata-700 104, India.
}

\begin{abstract}
In this paper we investigate the role played by dark energy in the form of Generalized cosmic Chaplygin gas in an accelerating universe described by FRW cosmology. We have tried to describe the model from the theoretical point of view of a field, by introducing a scalar field $\phi$ and a self interacting potential $V(\phi)$. The corresponding expressions for the field are obtained for the given model. Statefinder parameters have been used to characterize the dark energy model. Plots have been generated for characterizing different phases of universe diagrammatically and a comparative study is performed with the Modified Chaplygin gas model. As an outcome of the study, Generalized cosmic Chaplygin gas is identified as a much less constrained form of dark energy as compared to modified Chaplygin gas.
\end{abstract}

PACS numbers:

*Electronic address: prudra.math@gmail.com 


\section{INTRODUCTION}

At the turn of the last century a new feather was added to Edwin Hubble's discovery of the expanding universe, when it was confirmed from the observations of type Ia supernovae and CMBR data that the expansion is having an accelerating nature associated with it [1, 2]. It marked the beginning of a new era in cosmology, as it gave rise to the new concept of the mysterious dark energy(DE) [3]. DE, as the name suggests is an invisible form of energy, possessing a large negative pressure component, with the help of which it is able to bring about the cosmic acceleration. Hence this type of matter violates the strong energy condition, i.e., $\rho+3 p<0$.

Now, soon after this attribution, the entire cosmological society began to investigate for a suitable model that can effectively play the role of DE. The simplest form of DE was found to be a tiny cosmological constant, $\Lambda$, which gave rise to the simplest model of the accelerating universe, the $\Lambda \mathrm{CDM}$ model. DE associated with a scalar field [4] is called quintessence, and provides an effective model of the accelerating universe. As time rolled on various Chaplygin gas models came into existence. Extensive research saw Chaplygin gas (CG) [5] pave way to Generalized Chaplygin gas (GCG) 6] and Modified Chaplygin gas (MCG) [7, 8]. The MCG equation of state is given by

$$
p=A \rho-\frac{B}{\rho^{\alpha}}
$$

where $p$ and $\rho$ are respectively the pressure and energy density and $0 \leq \alpha \leq 1, A$ and $B$ are positive constants.

In 2003, P. F. Gonzalez-Diaz [9] introduced the Generalized cosmic Chaplygin gas (GCCG) model. The striking factor of the model, which made it unique, was that it can be made stable and free from unphysical behaviours even when the vacuum fluid enters the phantom era. In the previous studies related to DE corresponding to phantom era it was seen that Big-Rip was the final destiny, since the time derivative of scale-factor blows to infinity in finite time. For the first time P. F. Gonzalez-Diaz introducing the GCCG model showed that Big Rip singularity can easily be avoided. Hence in such models there is no requirement for evaporation of black hole to zero mass.

The equation of state of Generalized cosmic Chaplygin gas is,

$$
p=-\rho^{-\alpha}\left[C+\left\{\rho^{1+\alpha}-C\right\}^{-\omega}\right]
$$

where $C=\frac{A}{1+\omega}-1$, with A being a constant that can take on both positive and negative values, and $-L<\omega<0, L$ being a positive definite constant, which can take on values larger than unity.

As numerous DE models began appearing in the scene, it was of utmost necessity to devise a method that would both qualitatively and quantitatively discriminate between various DE models. 
The statefinder parameters [10] were introduced in this context by Sahni et al in 2003. These parameters are able to discriminate between different DE in a model independent manner. The diagnostic pair is constructed by the scale factor $a(t)$ and its derivatives as follows:

$$
r \equiv \frac{\dddot{a}}{a H^{3}} \quad s \equiv \frac{r-1}{3\left(q-\frac{1}{2}\right)}
$$

where $a$ is the scale factor of the universe, $H$ is the Hubble parameter, a dot (.) denotes differentiation with respect to the cosmic time $t$ and $q$ is the deceleration parameter given by,

$$
q=-\frac{\ddot{a}}{a H^{2}}
$$

In fact for $\Lambda \mathrm{CDM}$ model this pair take the values $r=1, s=0$. For any DE model the trajectory in the $r-s$ plane is obtained and the distance of this trajectory from the point $(1,0)$ is noted. This produces the required discrimination.

In section 2, all discussions are valid in general for open, closed and flat model of universe, i.e., for $k= \pm 1,0$. But in section 3, only the simple case of spatially flat universe $(k=0)$ is considered. In fact it is confirmed from various CMB experiments [11], that the flat model of universe follows spontaneously from the early inflationary phase, thus justifying our consideration.

In this work we have tried to describe the GCCG model from the theoretical point of view of a field, by introducing a scalar field $\phi$ and a self interacting potential $V(\phi)$ with the effective Lagrangian

$$
\mathcal{L}=\frac{1}{2} \dot{\phi}^{2}-V(\phi)
$$

In [6] it is shown that the flat Friedmann model with Chaplygin gas can be described equivalently in terms of a homogeneous minimally coupled scalar field $\phi$, by fitting the FRW equations for Chaplygin gas into Barrow's scheme [12]. The expressions for homogeneous scalar field $\phi(t)$ and potential $V(\phi)$ describing the Chaplygin cosmology was obtained by Kamenshchik et al [6, 13, 14]. In [15] the work was repeated considering MCG as the DE model. In this work we have obtained the corresponding expressions for GCCG model as a generalization of the previous works on Chaplygin gas cosmology.

The paper is organized as follows: In section 2 the expressions for $\phi(t)$ and $V(\phi)$ are obtained in terms of the scale factor $a(t)$ and the corresponding plots are generated to show how $V(\phi)$ varies as the scale factor varies. In section 3 , the evolution of the universe is studied in the $\{r, s\}$ plane for the entire physically realistic history of the universe and compared to that of the other Chaplygin gas models, especially MCG. In section 4, a complete graphical analysis is performed. In section 5 
a comparative study between MCG and GCCG is performed and finally the paper ends with some concluding remarks in section 6 .

\section{GENERALIZED COSMIC CHAPLYGIN GAS IN FRW COSMOLOGY}

The metric of a homogeneous and isotropic universe in the FRW model is

$$
d s^{2}=d t^{2}-a^{2}(t)\left[\frac{d r^{2}}{1-k r^{2}}+r^{2}\left(d \theta^{2}+\sin ^{2} \theta d \phi^{2}\right)\right]
$$

where $a(t)$ is the scale factor and $k(=0, \pm 1)$ is the curvature scalar.

The Einstein field equations are

$$
\frac{\dot{a}^{2}}{a^{2}}+\frac{k}{a^{2}}=\frac{1}{3} \rho
$$

and

$$
\frac{\ddot{a}}{a}=-\frac{1}{6}(\rho+3 p)
$$

where $\rho$ and $p$ are the energy density and the isotropic pressure, respectively. Here we choose $8 \pi G=c=1$.

The energy conservation equation is

$$
\dot{\rho}+3 \frac{\dot{a}}{a}(\rho+p)=0
$$

Using equation (2) in equation (9), we have the solution of $\rho$ as

$$
\rho=\left[C+\left\{1+\frac{B}{a^{3(1+\alpha)(1+\omega)}}\right\}^{\frac{1}{1+\omega}}\right]^{\frac{1}{1+\alpha}}
$$

where $B$ is an arbitrary integration constant.

Using eqns. (77, 8 and 10) we get the following differential equation,

$$
H^{2}=\frac{1}{3}\left[C+\left\{1+B(1+z)^{3(1+\alpha)(1+\omega)}\right\}\right]^{\frac{1}{1+\alpha}}-k(1+z)^{2}
$$

where $z$ is the redshift.

Now for small values of scale factor $a(t)$, we have,

$$
\rho \simeq \frac{B^{\frac{1}{(1+\omega)(1+\alpha)}}}{a^{3}}
$$


which is a very large value and corresponds to the universe dominated by an equation of state, $p=-\frac{1}{\rho^{Z}}$, where $Z=\alpha(1+\omega)+\omega$ is a constant.

For large values of scale factor $a(t)$, we have,

$$
\rho \simeq(C+1)^{\frac{1}{1+\alpha}}
$$

For this value of $\rho$, the value of $p$ is found to be,

$$
p \simeq-(C+1)^{\frac{1}{1+\alpha}}=-\rho
$$

which corresponds to an otherwise empty universe with a cosmological constant $(C+1)^{\frac{1}{1+\alpha}}$.

We know that an accelerating universe should have a negative deceleration parameter, $q$. So it is clear from the expression of deceleration parameter $\left(q=-\frac{\ddot{a}}{a H^{2}}\right)$ that, $\ddot{a}>0$. Some straightforward algebra using equations (8 and 10), gives,

$$
\left(1+\frac{B}{a^{3(1+\omega)(1+\alpha)}}\right)\left(1-2 C B^{-\frac{1}{1+\omega}} a^{3(1+\alpha)}\right)-3<0
$$

i.e.,

$$
X\left(1-2 C X^{-\frac{1}{1+\omega}}\right)<3
$$

where $X=1+\frac{B}{a^{3(1+\omega)(1+\alpha)}}$. Here the transition from decelerating to the accelerating universe occurs at $a=\left(\frac{8 C^{3}-1}{B}\right)^{\frac{1}{6}}$. So it is quite clear from eqn.(15) that for large values of $a$, the universe is accelerating whereas for smaller values of $a$, the universe is decelerating. Hence in order to be consistent with the cosmological observations we should consider large values of $a$ for our analysis. Considering the sub-leading terms in eqn. (10) we obtain for large values of scale factor, the following expressions for energy density and pressure:

$$
\rho \simeq C^{\frac{1}{1+\alpha}}+\frac{(Y-C)}{C^{\frac{\alpha}{1+\alpha}}(1+\alpha)}
$$

and

$$
p \simeq-\left[C^{\frac{1}{1+\alpha}}+\frac{(Y-C)}{C^{\frac{\alpha}{1+\alpha}}(1+\alpha)}\right]
$$

where $Y=C+\left\{1+\frac{B}{a^{3(1+\omega)(1+\alpha)}}\right\}^{\frac{1}{1+\omega}}$ 
Now we consider this energy density and pressure corresponding to a scalar field $\phi$ having a self-interacting potential $V(\phi)$. the Lagrangian of the scalar field is given by,

$$
L_{\phi}=\frac{1}{2} \dot{\phi}^{2}-V(\phi)
$$

The expressions for energy density $\rho_{\phi}$ and pressure $p_{\phi}$ for the scalar field are as follows,

$$
\rho_{\phi}=\frac{1}{2} \dot{\phi}^{2}+V(\phi)=\rho=\left[C+\left\{1+\frac{B}{a^{3(1+\alpha)(1+\omega)}}\right\}^{\frac{1}{1+\omega}}\right]^{\frac{1}{1+\alpha}}=Y^{\frac{1}{1+\alpha}}
$$

and

$$
\begin{gathered}
p_{\phi}=\frac{1}{2} \dot{\phi}^{2}-V(\phi)=-\left[C+\left\{1+\frac{B}{a^{3(1+\alpha)(1+\omega)}}\right\}^{\frac{1}{1+\omega}}\right]^{-\frac{\alpha}{1+\alpha}}\left[C+\left(1+\frac{B}{a^{3(1+\alpha)(1+\omega)}}\right)^{-\frac{\omega}{1+\omega}}\right] \\
=-Y^{-\frac{\alpha}{1+\alpha}}\left[C+(Y-C)^{-\omega}\right]
\end{gathered}
$$

For a flat universe, i.e., $k=0$, we have,

$$
\begin{aligned}
\dot{\phi}^{2}= & {\left[C+\left\{1+\frac{B}{\left.\left.a^{3(1+\alpha)(1+\omega)}\right\}^{\frac{1}{1+\omega}}\right]^{\frac{1}{1+\alpha}}}\left[1-\left\{C+\left\{1+\frac{B}{\left.\left.a^{3(1+\alpha)(1+\omega)}\right\}^{-\frac{\omega}{1+\omega}}\right\} \times}\right.\right.\right.\right.\right.} \\
& \left.\left\{C+\left\{1+\frac{B}{a^{3(1+\alpha)(1+\omega)}}\right\}^{\frac{1}{1+\omega}}\right\}^{-1}\right]=Y^{\frac{1}{1+\alpha}}\left[1-\frac{1}{Y\left\{C+(Y-C)^{-\omega}\right\}}\right]
\end{aligned}
$$

and

$$
\begin{array}{r}
V(\phi)=\frac{1}{2}\left[C+\left\{1+\frac{B}{a^{3(1+\alpha)(1+\omega)}}\right\}^{\frac{1}{1+\omega}}\right]^{\frac{1}{1+\alpha}}\left[1+\left\{C+\left\{1+\frac{B}{a^{3(1+\alpha)(1+\omega)}}\right\}^{-\frac{\omega}{1+\omega}}\right\} \times\right. \\
\left.\left\{C+\left\{1+\frac{B}{a^{3(1+\alpha)(1+\omega)}}\right\}^{\frac{1}{1+\omega}}\right\}^{-1}\right]=\frac{Y^{\frac{1}{1+\alpha}}}{2}\left[1+\frac{1}{Y\left\{C+(Y-C)^{-\omega}\right\}}\right]=Y^{\frac{1}{1+\alpha}}-\frac{1}{2} \dot{\phi}^{2}
\end{array}
$$




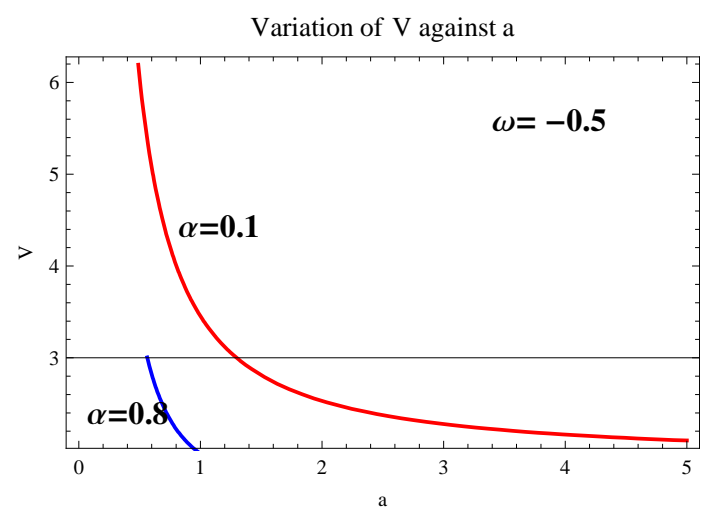

Fig.1

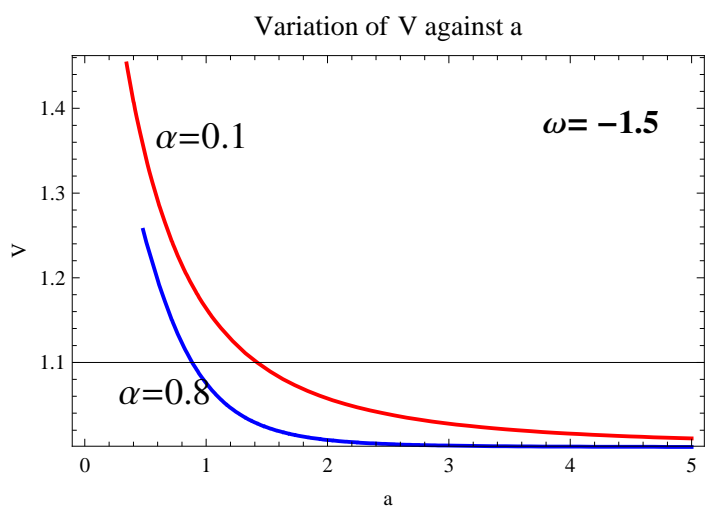

Fig.2

Figs.1: The potential is plotted against time for different values of $\alpha$. The values of $\alpha$ used are 0.1 and 0.8. The value of $\omega$ is taken as -0.5 .

Fig.2: The potential is plotted against time for different values of $\alpha$. The values of $\alpha$ used are 0.1 and 0.8 .

The value of $\omega$ is taken as -1.5 .

From the equation (21), we have the following relation between scale factor $a(t)$ and scalar field $\phi$,

$$
\phi=C^{\prime}+\int_{1}^{t} \sqrt{\left\{C+\left(1+B a v^{-3(1+w)(1+\alpha)}\right)^{\frac{1}{1+w}}\right\}^{\frac{1}{1+\alpha}}\left(1-\frac{C+\left(1+B a v^{-3(1+w)(1+\alpha)}\right)^{\frac{-w}{1+w}}}{C+\left(1+B a v^{-3(1+w)(1+\alpha)}\right)^{\frac{1}{1+w}}}\right)} d v
$$

where $C^{\prime}$ is the integration constant.

Now $2 V(\phi)=\rho-p=\rho-\frac{C+\left(\rho^{1+\alpha}-C\right)^{-\omega}}{\rho^{\alpha}}$.

\section{Case I:}

When $\quad \rho \rightarrow \infty$, i.e., $\quad a \rightarrow 0, \quad V(\phi) \rightarrow \infty$

\section{Case II:}

When $a \rightarrow \infty, \quad V(\phi) \rightarrow(C+1)^{\frac{1}{1+\alpha}}$

For example,

When $C=1 \quad$ and $\quad \alpha=1, \quad V(\phi) \rightarrow \sqrt{2} \quad$ as $\quad a \rightarrow \infty$

When $C=-1 \quad V(\phi) \rightarrow 0 \quad$ as $a \rightarrow \infty$

When $C=0 \quad V(\phi) \rightarrow 1 \quad$ as $a \rightarrow \infty$ 


\section{GEOMETRICAL DIAGNOSTIC USING STATEFINDER PARAMETERS}

The statefinder parameters are defined as follows,

$$
r \equiv \frac{\dddot{a}}{a H^{3}} \quad s \equiv \frac{r-1}{3(q-1 / 2)}
$$

where $H$ is the Hubble parameter and $q=-\frac{a \ddot{a}}{\dot{a}^{2}}$ is the deceleration parameter. Trajectories in the $r-s$ plane characterize different cosmological models. The $\Lambda$ CDM model corresponds to the fixed point $\{1,0\}$ in the $r-s$ plane. The deviations of the trajectories of different cosmological models from this point in the $r-s$ plane gives an effective comparison of the model from the standard model. So we proceed towards the statefinder analysis of the present model, GCCG.

For Friedmann model with a flat universe we have

$$
H^{2}=\frac{\dot{a}^{2}}{a^{2}}=\frac{1}{3} \rho
$$

and

$$
q=-\frac{\ddot{a}}{a H^{2}}=\frac{1}{2}+\frac{3}{2} \frac{p}{\rho}
$$

From the equation (24), we have,

$$
r=1+\frac{9}{2}\left(1+\frac{p}{\rho}\right) \frac{\partial p}{\partial \rho} \quad, \quad s=\left(1+\frac{\rho}{p}\right) \frac{\partial p}{\partial \rho}
$$

We get the ratio between $p$ and $\rho$ as,

$$
\frac{p}{\rho}=\frac{2(r-1)}{9 s}
$$

For GCCG the velocity of sound can be given as,

$$
v_{s}^{2}=\frac{\partial p}{\partial \rho}=-\frac{\alpha p}{\rho}+\frac{\omega(1+\alpha)}{\left(\rho^{1+\alpha}-C\right)^{\omega+1}}
$$

From equations (27), (28) and (29), we get the following relation between $r$ and $s$

$18 s^{2}(9 s)^{\frac{1}{\omega}}(r-1)+(9 s)^{1+\frac{1}{\omega}} 2 \alpha(r-1)+4 \alpha(9 s)^{\frac{1}{\omega}}(r-1)^{2}=\omega(1+\alpha)(2-2 r-9 C s)^{1+\frac{1}{\omega}}(9 s+2 r-2)$

Now we consider three different cases:

Case 1: When $\omega=-\frac{1}{2}$

For this case equation (30) becomes

$$
2\left[18 s^{2}(r-1)+18 \alpha(r-1) s+4 \alpha(r-1)^{2}\right](2-2 r-9 C s)+81 s^{2}(1+\alpha)(9 s+2 r-2)=0
$$


From the above equation it is quite clear that there is only one asymptote parallel to the $s$-axis, namely $r=1+\frac{9(1+\alpha)}{4 C}$. The asymptote intersects the curve at only one point

$$
\begin{gathered}
\left(1+\frac{9(1+\alpha)}{4 C},-\frac{A_{1}}{6 B_{1}}-\frac{\left(-A_{1}^{2}+12 B_{1} C_{1}\right)}{32^{2 / 3} B_{1} D_{1}+\sqrt{\left(D_{1}^{2}+4\left(-A_{1}^{2}+12 B_{1} C_{1}\right)^{3}\right)^{1 / 3}}}+\frac{1}{62^{1 / 3} B_{1}}\left\{D_{1}\right.\right. \\
\left.\left.+\sqrt{\left(D_{1}^{2}+4\left(-A_{1}^{2}+12 B_{1} C_{1}\right)^{3}\right)}\right\}^{1 / 3}\right)
\end{gathered}
$$

where,

$$
\begin{aligned}
& A_{1}=1-18 C+2 \alpha-18 C \alpha+4 C^{2} \alpha+\alpha^{2} \\
& B_{1}=\left(-17 C^{2}+C^{2} \alpha\right) \\
& C_{1}=\alpha+C^{2} \alpha+\alpha^{2}+C^{2} \alpha^{2} \\
& D_{1}=-2+108 C-1944 C^{2}+11664 C^{3}-12 \alpha+540 C \alpha-8412 C^{2} \alpha+46872 C^{3} \alpha-39600 C^{4} \alpha+ \\
& 11016 C^{5} \alpha-30 \alpha^{2}+1080 C \alpha^{2}-13560 C^{2} \alpha^{2}+58968 C^{3} \alpha^{2}-78648 C^{4} \alpha^{2}+23112 C^{5} \alpha^{2}-2448 C^{6} \alpha^{2}- \\
& 40 \alpha^{3}+1080 C \alpha^{3}-9648 C^{2} \alpha^{3}+23976 C^{3} \alpha^{3}-35976 C^{4} \alpha^{3}+11448 C^{5} \alpha^{3}-2432 C^{6} \alpha^{3}-30 \alpha^{4}+540 C \alpha^{4}- \\
& 2544 C^{2} \alpha^{4}+216 C^{3} \alpha^{4}+3000 C^{4} \alpha^{4}-648 C^{5} \alpha^{4}+144 C^{6} \alpha^{4}-12 \alpha^{5}+108 C \alpha^{5}+12 C^{2} \alpha^{5}-72 C^{4} \alpha^{5}-2 \alpha^{6}
\end{aligned}
$$

Case 2: When $\omega=-1$

For this case equation (30) reads

$$
2(r-1)\left[s+\alpha+\frac{2 \alpha(r-1)}{9 s}\right]+(1+\alpha)(9 s+2 r-2)=0
$$

Here the only asymptote parallel to the $s$-axis is $r=1-\frac{9(1+\alpha)}{2}$. Here the asymptote intersects the curve at the point $\left(1-\frac{9(1+\alpha)}{2}, \frac{\alpha(1+\alpha)}{2 \alpha+1}\right)$

\section{Case 3: When $\omega=-2$}

Here the equation (30) transform into

$$
9(1+\alpha)^{2} s(2-2 r-9 C s)(9 s+2 r-2)^{2}+\left[9 s^{2}(r-1)+9 \alpha(r-1) s+2 \alpha(r-1)^{2}\right]^{2}=0
$$

In this case we have two asymptotes parallel to $s$-axis, namely, $r=1 \pm 9 \sqrt{C}(1+\alpha)$. The asymptote $r=1+9 \sqrt{C}(1+\alpha)$ intersects the curve at the point

$$
\left(1+9 \sqrt{C}(1+\alpha), \frac{A_{2}}{6 B_{2}}-\frac{\left(-A_{2}^{2}+24 B_{2} C_{2}\right)}{\left[32^{2 / 3} B_{2}\left\{D_{2}+\sqrt{\left(D_{2}^{2}+4\left(-A_{2}^{2}+24 B_{2} C_{2}\right)^{3}\right)}\right\}^{1 / 3}\right]}+\frac{1}{62^{1 / 3} B_{2}}\left[D_{2}+\right.\right.
$$




$$
\left.\left.\sqrt{\left\{D_{2}^{2}+4\left(-A_{2}^{2}+24 B_{2} C_{2}\right)^{3}\right\}}\right]^{1 / 3}\right)
$$

where,

$$
\begin{aligned}
& A_{2}=8 \sqrt{C}+4 C^{3 / 2}+16 \sqrt{C} \alpha-4 C \alpha+8 C^{3 / 2} \alpha+7 \sqrt{C} \alpha^{2}-4 C \alpha^{2}+4 C^{3 / 2} \alpha^{2} \\
& B_{2}=1+2 C+\alpha-\sqrt{C} \alpha+2 C \alpha \\
& C_{2}=2 C+6 C \alpha+5 C \alpha^{2}+C \alpha^{3} \\
& D_{2}=128 C^{3 / 2}+1344 C^{5 / 2}+384 C^{7 / 2}-128 C^{9 / 2}+768 C^{3 / 2} \alpha-192 C^{2} \alpha+8064 C^{5 / 2} \alpha-192 C^{3} \alpha+ \\
& 2304 C^{7 / 2} \alpha+384 C^{4} \alpha-768 C^{9 / 2} \alpha+2016 C^{3 / 2} \alpha^{2}-960 C^{2} \alpha^{2}+20352 C^{5 / 2} \alpha^{2}-960 C^{3} \alpha^{2}+6624 C^{7 / 2} \alpha^{2}+ \\
& 1920 C^{4} \alpha^{2}-1920 C^{9 / 2} \alpha^{2}+2944 C^{3 / 2} \alpha^{3}-2160 C^{2} \alpha^{3}+27648 C^{5 / 2} \alpha^{3}-2848 C^{3} \alpha^{3}+11136 C^{7 / 2} \alpha^{3}+ \\
& 3840 C^{4} \alpha^{3}-2560 C^{9 / 2} \alpha^{3}+2520 C^{3 / 2} \alpha^{4}-2640 C^{2} \alpha^{4}+21672 C^{5 / 2} \alpha^{4}-4704 C^{3} \alpha^{4}+10944 C^{7 / 2} \alpha^{4}+ \\
& 3840 C^{4} \alpha^{4}-1920 C^{9 / 2} \alpha^{4}+1200 C^{3 / 2} \alpha^{5}-1728 C^{2} \alpha^{5}+9552 C^{5 / 2} \alpha^{5}-3744 C^{3} \alpha^{5}+5760 C^{7 / 2} \alpha^{5}+ \\
& 1920 C^{4} \alpha^{5}-768 C^{9 / 2} \alpha^{5}+250 C^{3 / 2} \alpha^{6}-480 C^{2} \alpha^{6}+1896 C^{5 / 2} \alpha^{6}-1120 C^{3} \alpha^{6}+1248 C^{7 / 2} \alpha^{6}+384 C^{4} \alpha^{6}- \\
& 128 C^{9 / 2} \alpha^{6}
\end{aligned}
$$

\section{GRAPHICAL ANALYSIS}

In the figures 1 and 2, the scalar field potential $V(\phi)$ is plotted against time for different values of the parameters involved. Two different plots are generated for different values of $\omega$. It is evident that the scalar field potential decreases as the scale factor increases. In an accelerating universe the scale factor increases gradually with time. So it is clear that the potential decreases with time. Plots with different values of $\alpha$ shows that the potential assumes larger values for smaller $\alpha$. On the contrary a comparison between figs. 1 and 2 indicate that with a decrease in the value of $\omega$, there is corresponding decrease in the value of potential.

In figure 3, the statefinder parameters $r$ and $s$ are plotted against each other for three different values of $\alpha$. It is seen that for $r=1$, all the three curves tend towards $s=0$, thus producing a correspondence with the $\Lambda \mathrm{CDM}$ model. With an increase in the value of $\alpha$, there is a gradual shift of the trajectories towards the right hand side of the plot, thus showing an increasing $r$ tendency. In this plot $C=2$ is kept constant throughout.

In Figure 4, the r-s trajectories are obtained for two different values of $C$, keeping $\alpha$ fixed. It is seen that for $C=6$ (red curve), $s=0$ more or less corresponds with $r=1$, whereas for $C=20$ (blue curve), $s=0$ does not exactly correspond to $r=1$, thus showing a greater deviation. Thus it can be concluded that as the value of $C$ increases, there is greater deviation of the GCCG model from the standard $\Lambda$ CDM model. Since $C$ can take both positive and negative 


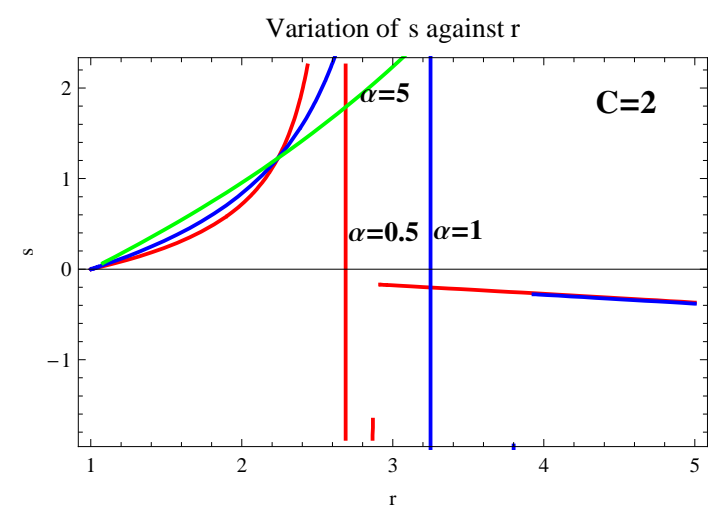

Fig. 3

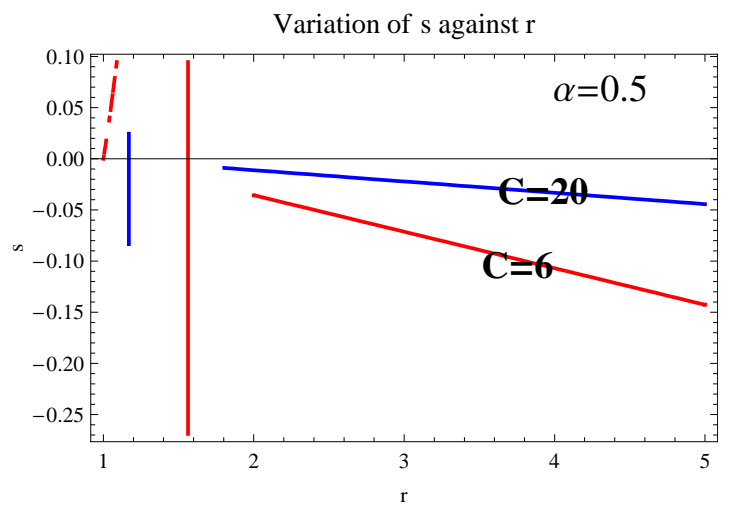

Fig. 4

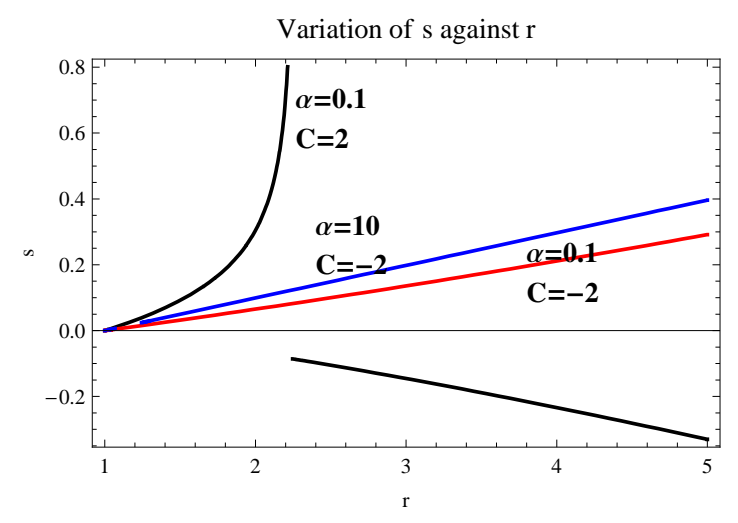

Fig. 5

Fig.3 : The statefinder parameters are plotted against each other for different values of $\alpha$. The values of $\alpha$ used are $0.5,1$ and 5 . The value of $C$ is taken as 2 .

Fig.4: The statefinder parameters are plotted agains each other for different values of $C$. The values of $C$ used are 6 and 20. The value of $\alpha$ is taken as 0.5 .

Fig.5 : The statefinder parameters are plotted against each other for different values of $C$ and $\alpha$. The values are furnished in the figure.

values, r-s plots for negative values of $C$ should be obtained and studied. This is accomplished in fig.5. Three different values of $\alpha$ are considered and the plots are generated.

\section{A COMPARATIVE STUDY BETWEEN THE ROLE OF GCCG AND MCG IN ACCELERATING UNIVERSE}

In [8] the authors studied the role of MCG in the accelerating universe. In this section we undergo a comparative study between this study and the study in [8]. We revisit the plots for the scalar field potential and statefinder parameters for MCG as witnessed in [8] in the figs. 6, 7, 8, 9 and 10. These plots when compared to the corresponding plots of GCCG exhibits a few similarities 
and dissimilarities. We study them separately below.

\section{A. Similarities}

We see that the plots 1 and 2 for GCCG almost show similar characteristics as the plots 6 and 7 for MCG, taking $A=1 / 3$. In these figures the potential decrease with increase in scale factor.

\section{B. Differences}

1) In the plots 8 and 9 for MCG, taking $A=1$, the curve behave quite differently from the plots 1 and 2 for GCCG. In case of MCG the potential increases steeply for a while before becoming stagnant at a particular value of $V$. But in case of GCCG there is a constant decrease in potential with increase in $a$, as seen before.

2) Fig 10 shows the $r-s$ plot for MCG. When compared with the $r-s$ plots for GCCG (figs. 3, 4 and 5), clear differences in trajectories are observed. This helps us to discriminate between the two DE models.

3) In fig. 10, the portion of the curve on the positive side of $s$, which is physically admissible is only the values of $r$ greater than $\left\{1+\frac{9}{2} A(1+A)\right\}$ (for $A=1 / 3$, it becomes equal to 3 ). Incidentally the portion of the curve between $r=1$ and $r=\left\{1+\frac{9}{2} A(1+A)\right\}$ on the positive side of $s$ is not admissible and that portion of the curve is not shown in [8]. The reason behind this is that in this restricted region constant $B$ becomes negative, which is inadmissible. Therefore the curve on the positive side of $s$ starts from the radiation era and goes asymptotically to the dust model, but the portion on the negative side of $s$ shows the evolution right from the dust state $(s=-\infty)$ to the $\Lambda \mathrm{CDM}$ model $(s=0)$. Hence the negative and the positive region complement each other, and the total curve (both positive $s$ and negative $s$ portions) represents the evolution of the universe from the radiation era to the $\Lambda \mathrm{CDM}$ model.

But in case of GCCG we see that there is no such restriction on any of the parameters $C, \omega$ and $\alpha$, since they do not have any restriction on either magnitude or sign. So the entire portion of the curves in figs. 3, 4 and 5 is physically admissible and there is no restricted portion, as it is in case of MCG. This is a striking difference! In fact it makes GCCG a much less constrained form of dark energy. 


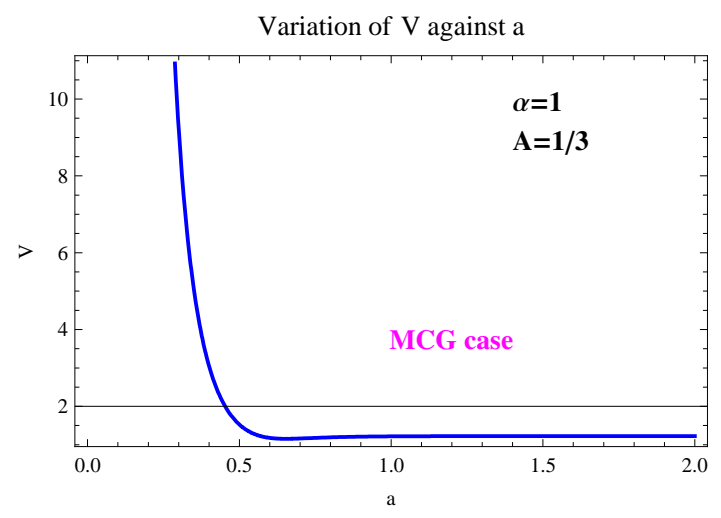

Fig. 6

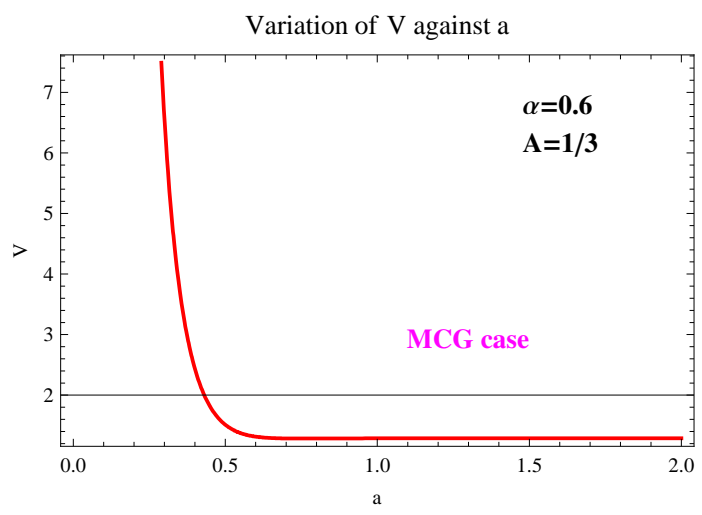

Fig. 7

Fig.6 : The field potential is plotted against the scale factor in case of Modified Chaplygin gas. The values of $\alpha$ and $A$ are respectively 1 and $1 / 3$.

Fig.7: The field potential is plotted against the scale factor in case of Modified Chaplygin gas. The values of $\alpha$ and $A$ are respectively 0.6 and $1 / 3$.

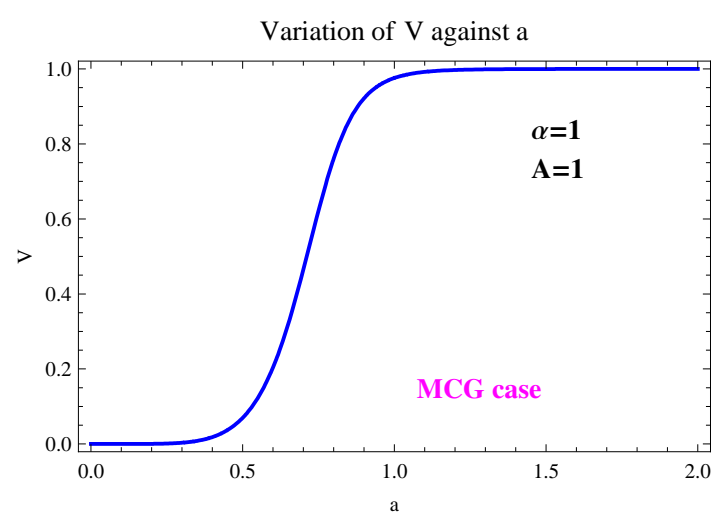

Fig. 8

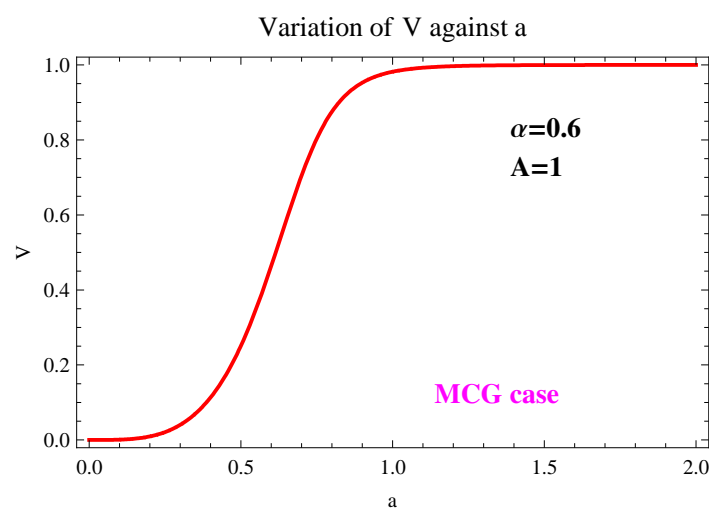

Fig. 9

Fig.8: The field potential is plotted against the scale factor in case of Modified Chaplygin gas. The values of $\alpha$ and $A$ are respectively 1 and 1.

Fig.9: The field potential is plotted against the scale factor in case of Modified Chaplygin gas. The values of $\alpha$ and $A$ are respectively 0.6 and 1 .

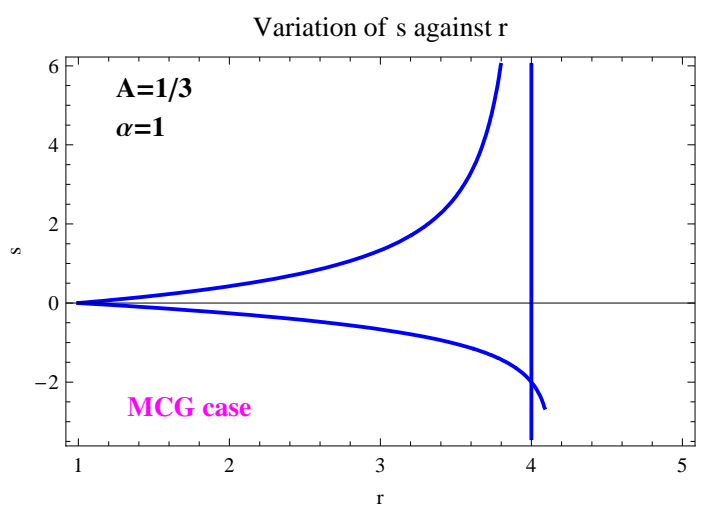

Fig. 10

Fig.10 : The statefinder parameters $r$ and s are plotted against each other in case of Modified Chaplygin gas. The values of $\alpha$ and $A$ are respectively 1 and $1 / 3$. 


\section{CONCLUSION}

In this work we have considered Generalized cosmic Chaplygin gas and tried to find the role played by it in an accelerating universe described by FRW cosmology. To accomplish this we have compared the GCCG model with a scalar field, and determined its potential. Plots were generated for the determined potential. Statefinder parameters were studied in detail in order to discriminate the DE model from other DE models. We have undergone a comparative study between the GCCG and MCG models as far as their role in the accelerating universe is concerned. The trajectories of the $r-s$ plots helps us to discriminate between the two models. For $A=1$, it was found that MCG shows an increase in the scalar potential in a certain region, as $a$ increases. But in case of GCCG, irrespective of whatever value is assigned to the parameters, the potential always show a downhill progress. It was witnessed that unlike MCG there is no restricted region in the $r-s$ plot for GCCG, thus making every portion of the curve physically admissible as it violates the admissible values of the B parameter of MCG. In case of GCCG there cannot be any such inadmissible region in the r-s plot. This is possible because the restrictions of the $C$, $\omega$ and $\alpha$ parameters of GCCG is much less pronounced than those of the parameters of MCG. - Hence GCCG is less constrained compared to MCG. This result is important because it gives a more universal character to GCCG as a dark energy. It is shown in [16] that GCCG as a dark energy is less effective than MCG and other dark energy models. In the background of this result our current finding of the universal nature of GCCG as a dark energy is quite unexpected! A dark energy suffering from much less restriction imposed by its parameters can be physically and mathematically more acceptable compared to those dark energy models which work under a very small domain with a lot of parameter constraints. It provides an independent outlook to the model capable of adapting itself to any domain of cosmology. Finally it should be stated that GCCG describes the universe from radiation era to $\Lambda \mathrm{CDM}$ era without constraining its parameters considerably.

[1] S. Perlmutter et al. :- Astrophys. J. 517, 565 (1999).

[2] D. N. Spergel et al. :- WMAP Collaboration, Astron. J. Suppl 148, 175(2003) [arXiv astro-ph/0302209]. 
[3] Riess, A. G. et al. :- [Supernova Search Team Collaboration], ApJ 607, 665(2004)[arXiv:0402512(astro-ph)].

[4] Nojiri, S., Odintsov, S.D. :- Phys. Rev. D 70 103522(2004).

[5] Kamenshchik, A., Moschella, U. and Pasquier, V. :- Phys. Lett. B 511, 265(2001).

[6] Gorini, V., Kamenshchik, A. and Moschella, U. :- Phys. Rev. D 67, 063509(2003).

[7] Benaoum, H. B.:- [arXiv:0205140(hep-th)].

[8] Debnath, U., Banerjee, A., Chakraborty, S. :- Class. Quantum. Grav. 21, 5609(2004).

[9] Gonzalez-Diaz, P. F. :- Phys. Rev. D 68021303 (R)(2003).

[10] Sahni, V., Saini, T. D., Starobinsky, A. A., Alam, U. :- JETP Lett. 77 201(2003).

[11] Benoit, A. et al :- Astron. Astrophys. 399, L25(2003).

[12] Barrow, J. D. :- Nucl. Phys. B 310 743(1988)

[13] Gorini, V., Kamenshchik, A., Moschella, U., and Pasquier, V. :- Phys. Rev. D 69 123512(2004)

[14] Gorini, V., Kamenshchik, A., Moschella, U., and Pasquier, V. :- Proc. 10th M Grossmann Meeting on Recent Developments in Theoretical and Experimental General Relativity, Gravitation and Relativistic Field Theories (MGXMMIII)(Rio de Janeiro, 20-26 July 2003) (Preprint gr-qc/0403062)

[15] Debnath, U., Banerjee, A., Chakraborty, S. :- Class. Quantum. Grav. 21, 5609(2004).

[16] Rudra, P. :- Astrophys. Space Sci.342 579 (2012) 